\title{
Chemical lysis of cyanobacteria
}

\author{
Kunal K. Mehta ${ }^{1}$, Niklaus H. Evitt ${ }^{2}$ and James R. Swartz ${ }^{1,2^{*}}$
}

\begin{abstract}
We have developed a mixture of enzymes and chemicals that completely lyse cyanobacteria. Since the treatment involves only readily-available chemicals and simple proteins that degrade the components of the cyanobacterial cell wall, it can easily be used in high-throughput applications requiring lysis for subsequent intracellular measurements. Our lysis technique consistently enables complete lysis of several different cyanobacterial strains, and we demonstrated that DNA, mRNA, and proteins are preserved in the lysates. Chemical lysis can be superior to existing techniques because of its convenience, reliability, and amenability to a variety of downstream applications.
\end{abstract}

\section{Background}

Significant efforts have been directed towards the fundamental goal of using photosynthesis to sustainably produce chemicals and fuels from sunlight and water, usually with either cyanobacteria or algae as hosts [1]. As the simplest photosynthetic organisms, cyanobacteria have attracted particular attention [2] for a range of applications including harvesting their remarkably wide library of natural products [3-5] and metabolic engineering for chemical production [6-10]. However, these advances have been limited by lower productivities in cyanobacteria than in traditional hosts. Two recent efforts to engineer cyanobacteria to produce chemicals by photosynthesis achieved productivities of $137 \mathrm{mg} / \mathrm{L} /$ day for isobutryaldehyde [11] and $391 \mathrm{mg} / \mathrm{L} /$ day for sucrose [12]. To our knowledge, these are the best productivities achieved from cyanobacteria in labscale cultures; however, they are almost two orders of magnitude lower than, for example, $14 \mathrm{~g} / \mathrm{L} /$ day for succinate in $E$. coli [13] and $3.6 \mathrm{~g} / \mathrm{L} /$ day for artemisinic acid in S. cerivisiae [14].

We believe high-throughput genetic engineering of cyanobacteria will be essential to overcome these limitations and enable the use of photosynthesis to sustainably synthesize chemicals. Applications for high-throughput genetic approaches include screening large libraries of parts [15] to optimize pathways and genetic mapping via insertional mutagenesis [16] to help determine genetic influences on observed phenotypes.

\footnotetext{
* Correspondence: jswartz@stanford.edu

'Department of Bioengineering, Stanford University, Stanford, CA 94305, USA ${ }^{2}$ Department of Chemical Engineering, Stanford University, Stanford, CA 94305, USA
}

Cell lysis to enable intracellular measurements is a fundamental requirement in the strain engineering cycle. It is particularly challenging in cyanobacteria compared to traditional hosts. A recent assessment of several existing methods for cyanobacterial lysis demonstrated effective lysis only by using low-throughput methods such as sonication, mechanical disruption, or lyophilization [17]. A controllable lysis system has also been developed wherein a lytic casette from a bacteriophage, including a lysozyme and accessory proteins, was expressed in Synechocystis sp. PCC 6803 under the control of a nickel-inducible promoter [18], but this requires prior genetic engineering and is not generally adaptable. Thus, new and convenient techniques amenable to high-throughput analysis are needed.

The major obstacle to lysis is the resiliance of the cell wall, which is thicker and more complex in cyanobacteria than in E. coli and many other commonly engineered organisms. The cyanobacterial cell wall contains a thicker and more highly crosslinked peptidoglycan layer [19], and cyanobacteria unlike E. coli have a surface layer ( $S$-layer) composed of polymerized proteins and an exopolysaccharide coating [20] (Fig. 1). The S-layer of Synechocystis, one of the most widely-used model cyanobacteria, has only recently been characterized in detail [21].

We sought to develop a simple, one-step chemical process that would degrade each of these barriers systematically. Some reagents for degrading cell wall components are well-established, such as lysozyme for peptidoglycan and detergents to permeabilize membranes. However, since other components of the cyanobacterial cell wall have not been well characterized, we tested a variety of other reagents to ensure all the layers of the cell wall could be breached. Here, we describe the cloning and 


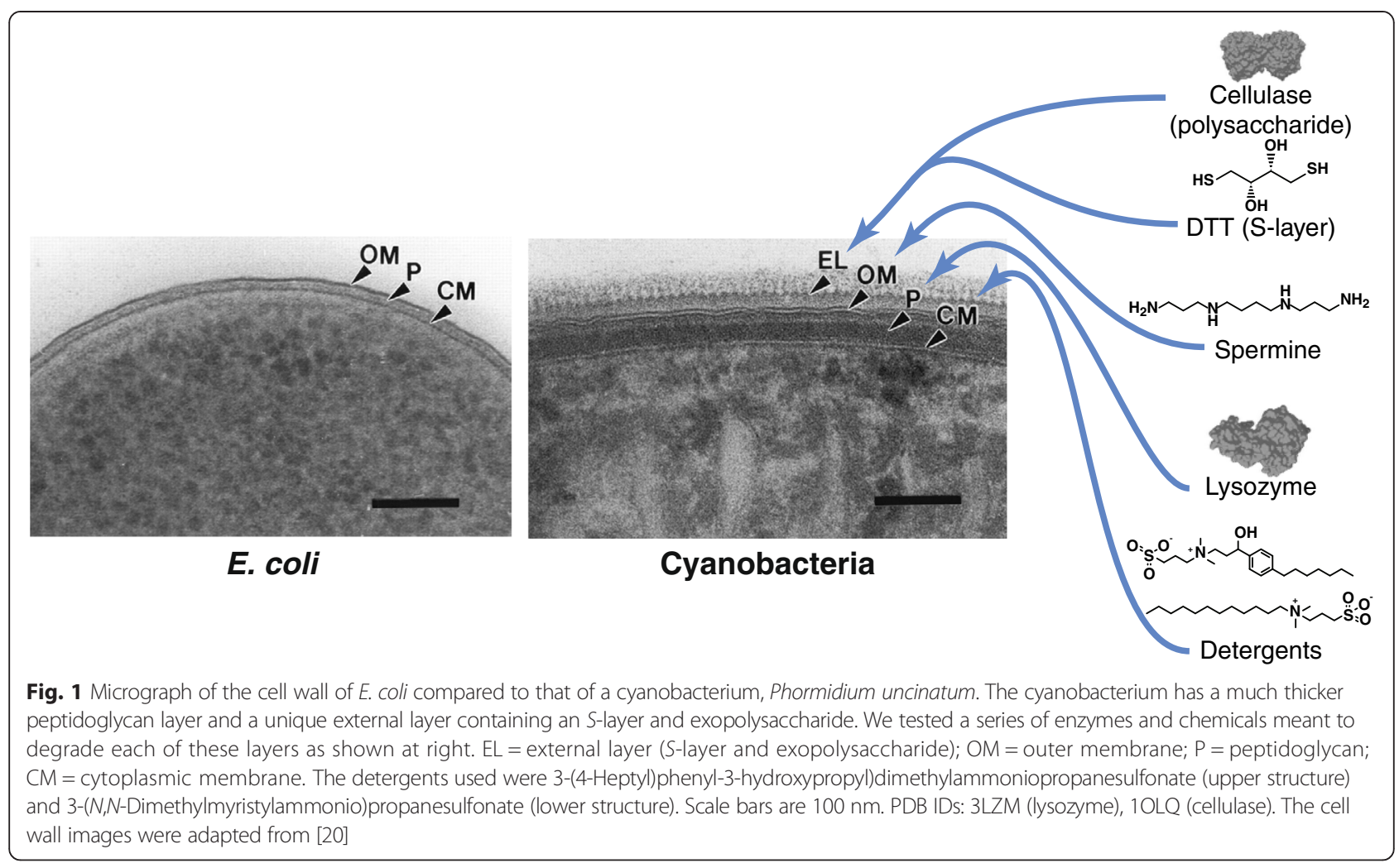

testing of a novel lysozyme from a cyanobacteria-specific bacteriophage and the development of a chemical treatment to fully lyse cyanobacteria in a high-throughput manner.

\section{Results}

We first tested a lysozyme from a cyanobacteria-specific phage [22] for its ability to more effectively degrade the cyanobacterial peptidoglycan layer relative to currently used enzymes. Our hypothesis was that a lysozyme from cyanobacterial phage might be uniquely suited to degrading the cyanobacterial peptidoglycan layer and might even be capable of breaking through other barriers as well. A novel lysozyme gene (GenBank accession number: KR259643), selected from a family of 23 identified by homology matching of ORFs in DNA from a cyanobacteria-phage community collected in Yellowstone National Park [22], was cloned and purified in E. coli.

We initially sought to optimize reaction conditions for cyanobacterial lysis. In these experiments, we compared a chemical lysis process using cyanophage lysozyme to a mechanical lysis procedure using vortexed glass beads as the best established procedure for cyanobacterial lysis. We tested a range of temperatures $\left(4-60{ }^{\circ} \mathrm{C}\right)$, reaction $\mathrm{pH}$ values (5.5-9.2), and reaction times (20-180 $\mathrm{min})$. In the initial tests, to achieve any lysis, we had to complement the lysozymes with BugBuster, a commercial lysis reagent which we have previously used with E. coli. Lysis was quantified by measuring the absorbance at $660 \mathrm{~nm}$ of chlorophyll released from the cells during the reaction; the "extent of lysis" is plotted in terms of raw absorbance units measured in the supernatant after centrifugation.

A treatment with lysozyme and BugBuster at $30-42{ }^{\circ} \mathrm{C}$ and $\mathrm{pH}$ 6-8 for $90 \mathrm{~min}$ achieved the best results, although it did not enable complete lysis (see Additional file 1). In these initial experiments, we estimated that the maximum extent of lysis corresponded to approximately $50 \%$ of the cells present, although the variability was significant.

A primary question was whether the new cyanophage lysozyme would perform better than other lysozymes against cyanobacteria. We tested this by comparing cyanophage lysozyme activity on Synechocystis sp. PCC 6803 to that of T4 lysozyme, a commercially available enzyme commonly used for E. coli lysis (Fig. 2). Overall, we found that cyanophage lysozyme was equivalent to T4 lysozyme in promoting cyanobacterial lysis (although at low lysozyme concentrations there was a slight advantage to using T4 lysozyme). Based on this, most subsequent experiments were done with T4 lysozyme.

Our initial tests revealed that lysozyme had to be supplemented with BugBuster to enable lysis. Since BugBuster is a proprietary mixture of unknown reagents, we sought to discover commercially available components that complement T4 lysozyme in breaching the outer barriers of cyanobacteria. Moreover, we found that BugBuster did not 


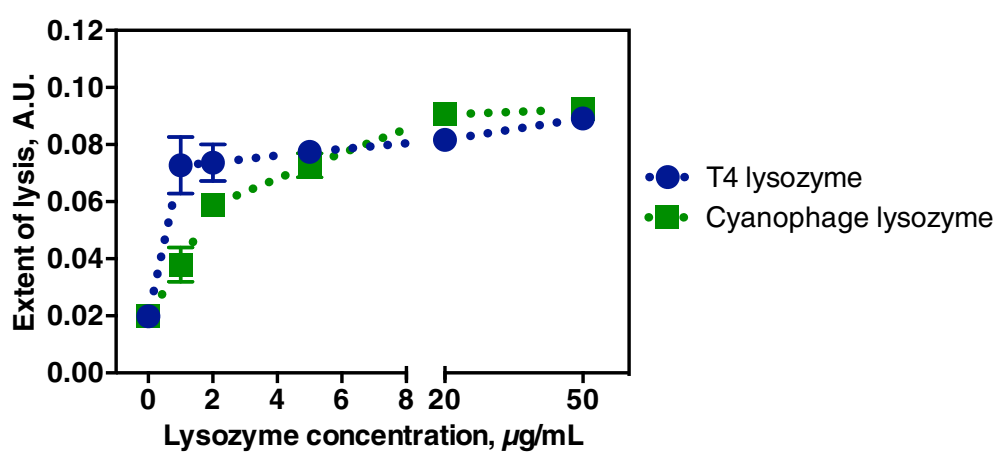

Fig. 2 Comparison of cyanophage lysozyme with T4 lysozyme for cyanobacterial lysis. The concentration of cyanophage lysozyme (purified in-house) and T4 lysozyme (MCLab) was titrated to compare their effectiveness in lysing Synechocystis sp. PCC 6803. Each reaction contained lysozyme at the indicated concentration, $25 \mathrm{mM} \mathrm{MES}$ buffer (pH 6.2), and 1 X BugBuster reagent. Reactions were incubated for 90 min at $37^{\circ} \mathrm{C}$. We estimated that the maximum lysis achieved here corresponded to $50 \%$ of total lysis; approx. $25 \%$ of the cells were lysed with BugBuster alone. Error bars are standard deviations of triplicate reactions

enable complete lysis under the optimized conditions mentioned above.

In addition to lysozyme, we tested cellulases (to break down exopolysaccharide), EDTA (to destabilize the outer membranes), DTT (to break potential disulfide bonds in the cell wall), spermine (which was previously shown to improve the activity of T4 phage lysozyme [23]), and the detergents 3-(4-Heptyl)phenyl-3-hydroxypropyl)dimethylammoniopropanesulfonate $(\mathrm{C} 7 \mathrm{Bz} 0)$ and $3-(\mathrm{N}, \mathrm{N}-$ Dimethyltetradecylammonio)propanesulfonate (SB3-14) to permeabilize membranes. These detergents were previously found to be particularly beneficial for cell lysis [24]. Further rationale for these components and their concentrations is given in Additional file 1. Figure 3a shows data from a series of reactions in which one of these components was omitted to determine which are beneficial for cyanobacterial lysis. In these and subsequent experiments, we achieved complete lysis, judged by the lack of any residual pigment/color in the debris pellet after incubation and centrifugation of the cell suspension (see Methods). Thus, these data are normalized to the signal from the reactions that gave complete lysis and are plotted in terms of "fraction of cells lysed".

Removing EDTA or cellulase had a minimal effect on lysis efficiency, but spermine and DTT were both important, with less than $50 \%$ lysis activity without either. EDTA was originally included to help destabilize the outer membrane by chelating divalent cations that stabilize negatively-charged sugars; its lack of effect in both Synechocystis (Fig. 3a) and E. coli (Additional file 1) suggests that the detergents by themselves enable sufficient permeabilization of the membranes.

Removing cellulase, which was included to degrade the secreted exopolysaccharide layer, also had a minimal effect on lysis efficiency. This suggests that exopolysaccharide is not a significant barrier to lysozyme and the other additives. However, these experiments were performed on cells in exponential phase, and we suspected that exopoysaccharide would be thicker in stationary phase. Thus we also tested the effect of cellulase on late-stage cultures. Even in this case, cellulase did not increase the extent of lysis (Additional file 1), suggesting the EPS in general does not pose a significant barrier to other components. Based on these results, subsequent experiments were performed with T4 lysozyme, detergents, spermine, and DTT.

We next performed a design-of-experiments (DOE) analysis to optimize the concentrations of these four reagents (Fig. $3 \mathrm{~b}$ ) and potentially discover interactions between cyanophage lysozyme, detergents, spermine, and DTT. It was interesting that several of the combinations tested gave virtually complete lysis. We thought this might suggest an interaction between some of the components in the mixture, but the DOE analysis did not reveal any significant interactions. Marginal means analysis of the DOE results predicted that the optimal combination is $50 \mu \mathrm{g} / \mathrm{mL}$ cyanophage lysozyme, $2 \mathrm{X}$ detergents, $400 \mu \mathrm{M}$ spermine, and $200 \mathrm{mM}$ DTT; subsequent experiments were done with this cocktail, except that $10 \mu \mathrm{g} / \mathrm{mL}$ T4 lysozyme was used instead of $50 \mu \mathrm{g} / \mathrm{mL}$ cyanophage lysozyme based on a separate optimization for that component (see Additional file 1).

Since the requirement for DTT at such a high concentration was particularly interesting, we titrated it separately while keeping the concentrations of the other components constant (Fig. 3c). This confirmed the results of the designof-experiments, with a significant increase in lysis activity even from 100 to $200 \mathrm{mM}$. We examine the implications further in the Discussion.

To show the utility of our procedure with additional strains of cyanobacteria, we tested lysis of Synechococcus sp. PCC 7942, another widely-used model strain, and Synechococcus JA-2-3B'a, a strain isolated from a Yellowstone hot spring [22] (Fig. 4). Full lysis was obtained with each strain using the cocktail. Furthermore, comparison 

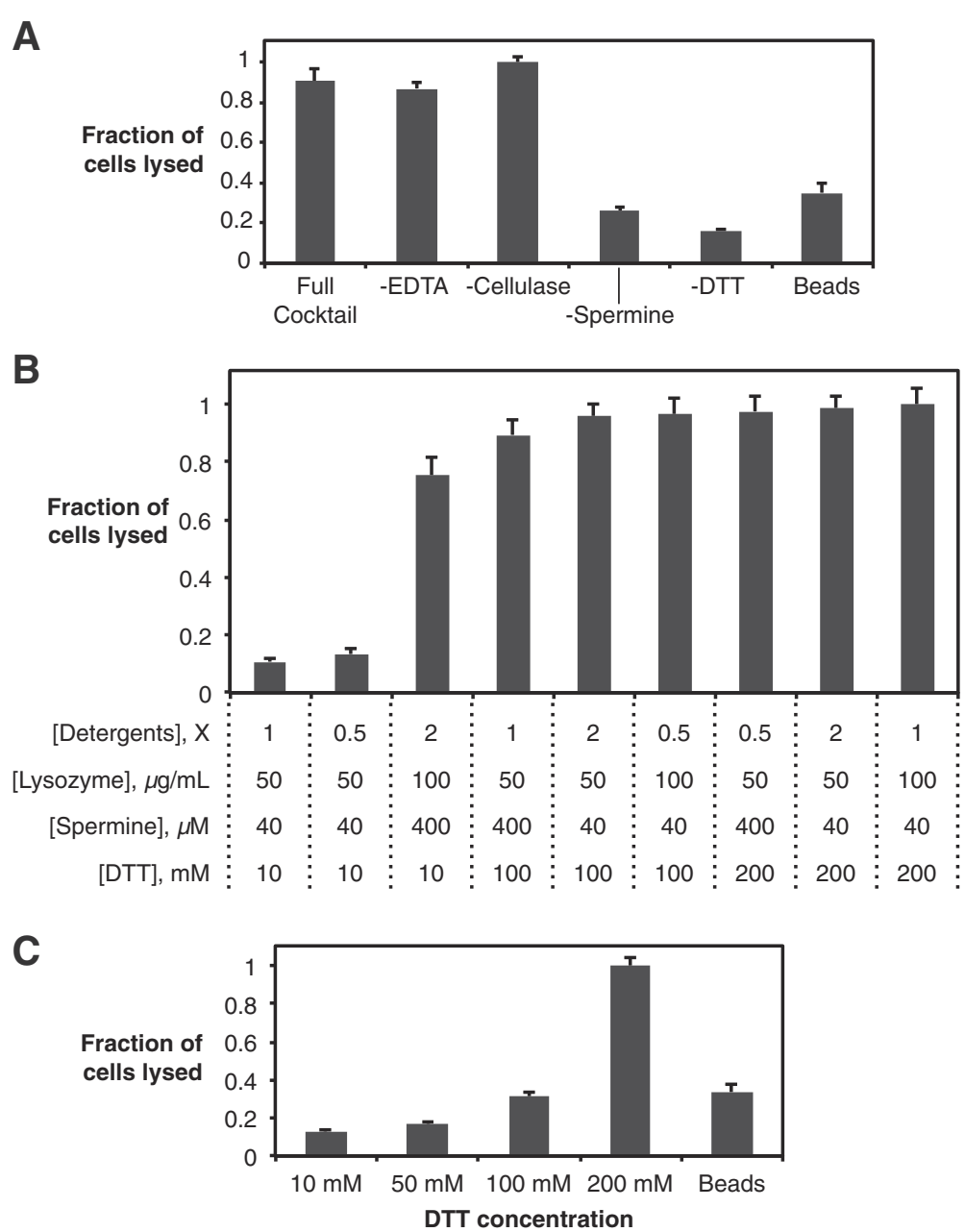

Fig. 3 A chemical cocktail for cyanobacterial lysis. All reactions were shaken for 90 min at $37^{\circ} \mathrm{C}$. a Testing the contribution of EDTA, cellulase, spermine, and DTT to cell lysis. All samples contained T4 lysozyme at $10 \mu \mathrm{g} / \mathrm{mL}$ and the detergents C7BzO (0.1 \% w/v) and SB3-14 (1 \% w/v). The "full cocktail" also contained EDTA at $1 \mathrm{mM}$, T. viride cellulase at $50 \mu \mathrm{g} / \mathrm{mL}$, spermine at $40 \mu \mathrm{M}$, and DTT at $200 \mathrm{mM}$; other samples omitted one of the reagents as indicated. Error bars are standard deviations of three independent experiments. $\mathbf{b}$ A design-of-experiments optimization of the levels of lysozyme, detergent, spermine, and DTT. The lysozyme was cyanophage lysozyme in this experiment. "1 X detergents" refers to 1 \% C7BzO and $0.1 \%$ SB3-14 (w/v). Error bars are standard deviations of triplicate reactions. c Influence of DTT concentration with other components as described for the "full cocktail" in (A), omitting EDTA and cellulase. Error bars are standard deviations of three independent experiments

with the commonly used bead lysis procedure indicated that our new chemical procedure can provide two- to three-fold improvement in lysis and more reliably enables full lysis of these three strains, highlighting the observation that mechanical disruption can be an unreliable method for cyanobacterial lysis.

We demonstrated the biological utility of lysates produced with our chemical cocktail in several ways. It is important that a lysis procedure preserve the activity of enzymes in the lysate. We chose ferredoxin-NADP ${ }^{+}$reductase as an example enzyme, and demonstrated that its activity is preserved in our lysate using a cytochrome $c$ reduction assay [25] (Fig. 5, top); the activity was comparable to that from a lysate produced by bead disruption.
We also amplified an 800 bp genomic fragment via PCR, showing that genomic DNA was not degraded in our lysates (Fig. 5, bottom). Finally, we isolated mRNA (Quick-RNA miniprep kit, Zymo) and reverse-transcribed cDNA (Verso reverse-transcription kit, Thermo-Fisher) with comparable yields starting from lysates produced with our cocktail or with beads (not shown). These results suggest that our chemical cocktail preserves biological components in cyanobacterial lysates for a variety of applications.

\section{Discussion}

Our chemical treatment uses detergents to permeabilize membranes in the cyanobacterial cell wall, spermine and 


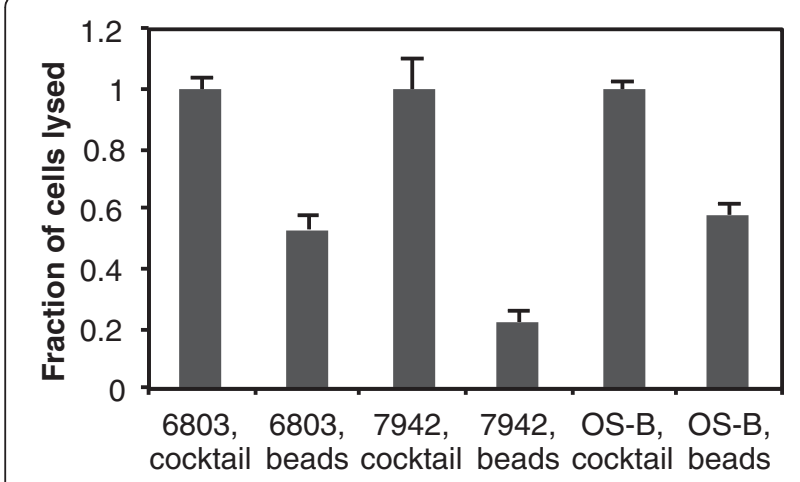

Fig. 4 Lysis of Synechocystis sp. PCC 6803, Synechococcus sp. PCC 7942, and Synechococcus JA-2-3B'a ("OS-B"). The "cocktail" contained T4 lysozyme at $10 \mu \mathrm{g} / \mathrm{mL}$, the detergents C7BzO (0.2\% w/v) and SB3-14 ( $2 \% \mathrm{~W} / \mathrm{v})$, spermine at $400 \mu \mathrm{M}$, and DTT at $200 \mathrm{mM}$; "beads" refers to vortexing with glass beads (see Methods). Error bars are standard deviations of triplicate samples

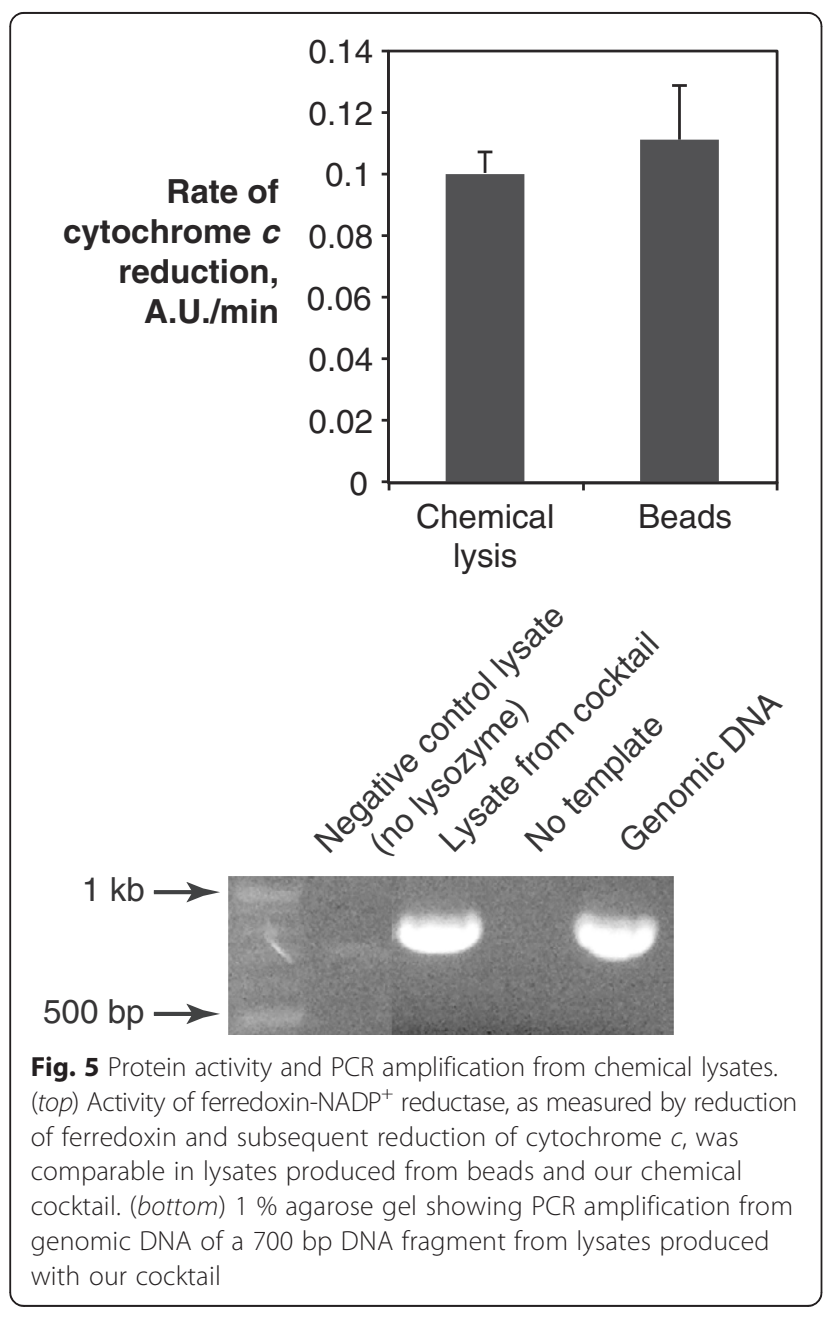

DTT to weaken the external layer, and lysozyme to break down peptidoglycan. Initially, we thought a lysozyme from a cyanobacteria-specific phage might be uniquely suited to lysing cyanobacteria, but this was not the case.

Lysozymes cleave the linkage between $\mathrm{N}$-acetylmuramic acid and $\mathrm{N}$-acetylglucosamine in peptidoglycan, which is consistent across almost all bacteria. This could explain why T4 lysozyme worked as well as cyanophage lysozyme against cyanobacteria: Although the cyanobacterial peptidoglycan is thicker and more highly cross-linked than in other species, the actual target of the lysozyme is the sugar chain sequence, which is similar. Nevertheless, the overall cyanobacterial cell wall clearly presents a unique challenge, and a systematic approach was required to breach it.

DTT was the most surprising additive required for full lysis (Fig. 3c). It is generally used as a reducing agent. We initially thought the target might be disulfide bonds in the S-layer, but the Synechocystis S-layer protein has no cysteine residues [21]. Certain cell wall components in yeast are disulfide bonded to each other [26]; it is possible that similar disulfide bonds exist between other components of the Synechocystis cell wall - for example, between membrane proteins, particularly in the outer membrane. T4 lysozyme does not contain disulfide bonds [27]; combined with the fact that DTT does not significantly promote $E$. coli lysis, we do not believe DTT acts on the lysozyme. Moreover, the concentration of DTT that was required - at least $50 \mathrm{mM}$, with up to $200 \mathrm{mM}$ for the full benefit - is far higher than that typically needed to reduce disulfide bonds. We note that the results of the DTT titration (Fig. 3c) indicating a requirement for $200 \mathrm{mM}$ DTT seem to disagree with some of the individual conditions in the design-ofexperiments (Fig. 3b). However, the requirement for $200 \mathrm{mM}$ DTT was consistently reproducible over several independent experiments.

We considered the possibility that the requirement for a high concentration of DTT implies a physical effect, perhaps an intercalation in one of the layers of the cell wall, rather than a chemical effect. We tested this by substituting DTT (redox potential of $-330 \mathrm{mV}$ at $\mathrm{pH}$ 7) with $\beta$-Mercaptoethanol (redox potential of $-260 \mathrm{mV}$ ) and TCEP (a stronger reducing agent than DTT). $\beta$ Mercaptoethanol was essentially as effective as DTT. TCEP also effectively contributed to cyanobacterial lysis, but the lysates had a red color instead of the yellow-green color normally seen; this decreased the absorbance measured at $660 \mathrm{~nm}$ and suggests that TCEP may reduce other molecules in the lysate, perhaps the pigments (data in Additional file 1). The fact that other reducing agents could replace DTT suggests that the function of DTT in the cocktail is indeed a reducing one (as opposed to a physical effect); further 
work is needed to determine why such a high concentration is required.

Spermine was also required for complete cyanobacterial lysis. Previous work with T4 lysozyme against E. coli [23] suggested that spermine directly enhances the muramidase activity of T4 lysozyme at spermine concentrations below $100 \mu \mathrm{M}$, and stabilizes spheroplasts at higher concentrations, counteracting that enhancement. However, we achieved virtually complete lysis of $E$. coli with lysozyme and detergent only, with small additional benefits from either EDTA or DTT. Since spermine is required for lysis of cyanobacteria but not E. coli using our cocktail, we believe its benefit here is through an interaction with a unique feature of the cyanobacterial cell wall and not with lysozyme. At our reaction $\mathrm{pH}$, spermine is fully protonated and carries four positive charges, so it probably does not pass through either cell membrane; most likely, it acts on the exopolysaccharide or S-layer.

To verify biological activity in lysates produced by our cocktail, we tested enzyme activity (using ferredoxinNADPH reductase as an example enzyme), DNA amplification by PCR, and isolation and reverse transcription of mRNA. We quantified lysis by measuring the release of pigments into the lysate; this necessarily means that the pigments were not damaged by the lysis cocktail. For proteins in particular, adsorption onto the large surface area presented by glass beads can be a problem, so a chemical procedure could provide a significant advantage for protein analysis. We note a potential limitation of our procedure in that the high concentration of DTT could break disulfide bonds in proteins.

At the scales we have used (reaction volumes $100 \mu \mathrm{L} ; \sim 1 \mu \mathrm{g}$ cells), we found that chemical lysis was significantly more effective than mechanical disruption with beads. We believe this is because mechanical disruption, especially for cells with such a strong cell wall, requires tremendous force often only provided by specialized equipment such as a BeadBeater homogenizer.

\section{Conclusions}

We examined a fundamental operation in the strain engineering cycle, cell lysis, from the perspective of the unique cyanobacterial cell wall. As a result, we developed a chemical treatment that fully lyses several strains of cyanobacteria at physiologically relevant conditions and does so more effectively than mechanical disruption with glass beads.

Our optimized cocktail consists of T4 lysozyme at $10 \mu \mathrm{g} /$ $\mathrm{mL}$, the detergents $\mathrm{C} 7 \mathrm{BzO}$ at $0.2 \% \mathrm{w} / \mathrm{v}$ and $\mathrm{SB} 3-14$ at $2 \% \mathrm{w} / \mathrm{v}$, spermine at $400 \mu \mathrm{M}$, and DTT at $200 \mathrm{mM}$. This reliably enabled full lysis of three different cyanobacterial strains.

Our lysis procedure uses only chemical components, making it easily adaptible to high-throughput applications, and preserves genomic DNA, mRNA, and enzyme activity for further analysis. We believe this will expand the scope of intracellular measurements that are feasible in cyanobacteria.

\section{Methods}

\section{Strains and plasmids}

DNA fragments were joined by Gibson assembly [28] and propagated in E. coli strain DH5 $\alpha$, and proteins were expressed in E. coli strain BL-21 (DE3). Plasmids for in vivo expression use a pET21b backbone (Novagen), and plasmids for expression by cell-free protein synthesis use a pY71 backbone [29] modified with an XbaI restriction site between $\mathrm{P}_{\mathrm{T} 7}$ and the ribosome-binding site to allow for modification of the region coding for the $5^{\prime}$ end of the mRNA. In all constructs, the sequence of this region, starting 25 bp upstream of the RBS and extending through the first $15 \mathrm{bp}$ of the coding sequence, was optimized for the mRNA to have a folding energy $\Delta G_{\mathrm{f}}>-1.5 \mathrm{kcal} / \mathrm{mol}$ (using a custom MATLAB script, optimizeForCFPS.m, available at https://gist.github.com/kkmehta/0a1dbe272d7 ff2aed011\#file-optimizeforcfps-m) to minimize inhibition of translation initiation by $5^{\prime}$ secondary structure. Table 1 contains a list of plasmids constructed for this work. The gene sequence for cyanophage lysozyme 1 has been deposited in GenBank with accession number KR259643.

\section{Protein expression and purification}

Proteins were expressed in vivo from BL21 (DE3) strains using IPTG induction. Briefly, $1 \mathrm{~L}$ cultures in terrific broth (Invitrogen) supplemented with ampicillin at $100 \mu \mathrm{g} / \mathrm{mL}$ were grown at $37{ }^{\circ} \mathrm{C}$ to OD $\sim 1.5$ and induced with IPTG at $100 \mu \mathrm{M}$. At this point, the temperature was reduced to $25^{\circ} \mathrm{C}$ and proteins were expressed overnight. Cultures were harvested by centrifugation at $3000 \times \mathrm{g}$ and cells were lysed in a single pass through a high-pressure homogenizer

Table 1 Plasmids constructed for this work

\begin{tabular}{ll}
\hline Plasmid & Backbone::insert \\
\hline pKM011 & pET21b::cpL1 \\
pKM012 & pY71X::cpL1 \\
pKM013 & pET21b::cpL2 \\
pKM014 & pY71X::cpL2 \\
pKM015 & pET21b::cpL3 \\
pKM016 & pY71X::cpL3 \\
pKM024 & pET21b::strepll-GSA-cpL1 \\
pKM025 & pET21b::cpL1-GSA-strepll \\
\hline
\end{tabular}

The lysozyme gene used in this study was "cyanophage lysozyme 1" (cpL1; sequence provided in Additional file 1). Two other genes, $\mathrm{cpL} 2$ and $\mathrm{cpL}$, were also cloned, but did not express solubly in $E$. coli. The genes were cloned into either the $\mathrm{pET} 21 \mathrm{~b}$ vector for in vivo expression or the $\mathrm{pY71X}$ vector for cell-free expression (data not shown) 
(Avestin) at $>18,000$ PSI. Debris and insoluble material were removed by centrifugation at $23,000 \times \mathrm{g}$. The soluble supernatant was purified on a streptactin column (IBA Life Sciences) according to the manufacturer's protocol. After purification, elution fractions were pooled, concentrated $\sim 30 \mathrm{X}$ using a centrifugal filter device (Amicon Ultra, $10 \mathrm{kDa}$ cutoff, Millipore), formulated in $20 \%$ sucrose, flash frozen in liquid nitrogen, and stored at $80^{\circ} \mathrm{C}$. Typical yields were $1 \mathrm{mg}$ of purified protein from 3 (wet) grams of cells.

\section{Cyanobacterial lysis reaction}

Unless otherwise stated, lysis reactions were carried out on Synechocystis sp. PCC 6803 (ATCC 27184) cells grown in BG-11 medium (Sigma) under constant illumination from fluorescent lamps at $\sim 200 \mu \mathrm{E} / \mathrm{m}^{2} / \mathrm{s}$ while sparging a mixture of $5 \% \mathrm{CO}_{2}$ in air. Cells were harvested in exponential phase (culture $\mathrm{OD}_{750}$ of $0.5-1$; doubling time 10-15 h). For lysis experiments, cells were pelleted by centrifugation and resuspended to OD 1.5 in MES buffer at pH 6.2 with lysozyme and either the specified lysis components (see Results) or $1 \mathrm{X}$ BugBuster protein extraction reagent (Novagen). Reactions were conducted in either microcentrifuge tubes $(300 \mu \mathrm{L}$ reactions, shaken on a vortexer (vortex genie, VWR)) or deep-well 96-well plates (100-200 $\mu \mathrm{L}$ reactions, shaken on a Thermo Scientific titer plate shaker (shake setting 7)). Wells in the plate were sealed with a custom PDMS gasket to prevent cross-contamination while shaking. After the reaction, insoluble debris were removed by centrifugation at $10,000 \times \mathrm{g}$ for $5 \mathrm{~min}$, and the degree of lysis was quantified by measuring absorbance of the supernatant at $660 \mathrm{~nm}$, corresponding to a chlorophyll absorbance peak. Total lysis was confirmed by the absence of green color in the debris pellets, indicating that all cells had lysed and released their chlorophyll into the supernatant. Mechanical lysis via glass beads (150-200 $\mu \mathrm{M}$ in diameter; Sigma-Aldrich) was used as a comparison in many experiments, as this is the current standard for cyanobacterial lysis. In these reactions, beads at $25 \%$ of the reaction volume [30, 31] were added to the cell suspension in buffer and detergents (to solubilize chlorophyll); these reactions were incubated and otherwise processed as described above.

All chemicals were obtained from Sigma-Aldrich. T4 lysozyme was obtained from MClab. Cellulases from Trichoderma viride, Trichoderma reesei, and Aspergillus niger (Sigma) were a kind donation from Lynette Cegelski. Design-of-experiments calculations were done in SPSS (IBM). The orthogonal design function was used to generate the set of experimental conditions, and a univariate general linear model was used to analyze the results.

\section{Additional file}

Additional file 1: Coding sequence of cyanophage lysozyme, rationale for cocktail components and concenetrations, and results of additional optimization experiments.

\section{Competing interests}

The authors declare that they have no competing interests.

\section{Authors' contributions}

KKM and NHE performed experiments; KKM, NHE, and JRS analyzed results. KKM and JRS wrote the manuscript, and all authors read and approved the final version.

\section{Acknowledgements}

We gratefully acknowledge Devaki Bhaya and Michelle Davison for the gene sequences of cyanophage lysozymes and a sample of Synechocystis OS-B, Rick Zuzow and the Bhaya group for many stimulating discussions, and the Bio-X Interdisciplinary Initiatives Program at Stanford for funding. KKM was also supported by a DOE Office of Science Graduate Fellowship, a Siebel Scholarship, and an ARCS Fellowship.

Received: 17 March 2015 Accepted: 19 May 2015

Published online: 05 June 2015

\section{References}

1. Desai SH, Atsumi S. Photosynthetic approaches to chemical biotechnology. Curr Opin Biotech. 2013;24:1031-6.

2. Ducat DC, Way JC, Silver PA. Engineering cyanobacteria to generate high-value products. Trends Biotechnol. 2011;29:95-103.

3. Kehr J-C, Gatte Picchi D, Dittmann E. Natural product biosyntheses in cyanobacteria: a treasure trove of unique enzymes. Beilstein J Org Chem. 2011;7:1622-35.

4. Welker M, Dittmann E, von Döhren $H$. Cyanobacteria as a source of natural products. Methods Enzymol. 2012;517:23-46.

5. Vestola J, Shishido TK, Jokela J, Fewer DP, Aitio O, Permi P, et al. Hassallidins, antifungal glycolipopeptides, are widespread among cyanobacteria and are the end-product of a nonribosomal pathway. PNAS. 2014;111:E1909-17.

6. Oliver JWK, Machado IMP, Yoneda H, Atsumi S. Cyanobacterial conversion of carbon dioxide to 2,3-butanediol. PNAS. 2013;110:1249-54.

7. Ungerer J, Tao L, Davis M, Ghirardi M, Maness P-C, Yu J. Sustained photosynthetic conversion of $\mathrm{CO} 2$ to ethylene in recombinant cyanobacterium Synechocystis 6803. Energy Environ Sci. 2012;5:8998.

8. Tyo KEJ, Jin Y-S, Espinoza FA, Stephanopoulos G. Identification of gene disruptions for increased poly-3-hydroxybutyrate accumulation in Synechocystis PCC 6803. Biotechnol Prog. 2009;25:1236-43.

9. Liu X, Fallon S, Sheng J, Curtiss R. CO2-limitation-inducible Green Recovery of fatty acids from cyanobacterial biomass. PNAS. 2011;108:6905-8.

10. Angermayr SA, Paszota M, Hellingwerf KJ. Engineering a cyanobacterial cell factory for production of lactic acid. Appl Environ Microbiol. 2012;78:7098-106.

11. Atsumi S, Higashide W, Liao JC. Direct photosynthetic recycling of carbon dioxide to isobutyraldehyde. Nat Biotechnol. 2009;27:1177-80.

12. Ducat DC, Avelar-Rivas JA, Way JC, Silver P. A. Re-routing Carbon-flux to Enhance Photosynthetic Productivity. Appl Environ Microbiol. 2012;78:2660-8.

13. Millard CS, Chao YP, Liao JC, Donnelly MI. Enhanced production of succinic acid by overexpression of phosphoenolpyruvate carboxylase in Escherichia coli. Appl Environ Microbiol. 1996;62:1808-10.

14. Paddon CJ, Westfall PJ, Pitera DJ, Benjamin K, Fisher K, McPhee D, et al. High-level semi-synthetic production of the potent antimalarial artemisinin. Nature. 2013:496:528-32.

15. Wang HH, Isaacs FJ, Carr PA, Sun ZZ, Xu G, Forest CR, et al. Programming cells by multiplex genome engineering and accelerated evolution. Nature. 2009;460:894-8.

16. Kufryk GI, Sachet M, Schmetterer G, Vermaas WFJ. Transformation of the cyanobacterium Synechocystis sp. PCC 6803 as a tool for genetic mapping: optimization of efficiency. FEMS Microbiol Lett. 2002;206:215-9.

17. Kim IS, Nguyen G-H, Kim S-Y, Lee J-W, Yu H-W. Evaluation of methods for cyanobacterial cell lysis and toxin (microcystin-LR) extraction using chromatographic and mass spectrometric analyses. Environ Eng Res. 2009;14:250-4. 
18. Liu X, Curtiss R. Nickel-inducible lysis system in Synechocystis sp. PCC 6803. PNAS. 2009;106:21550-4.

19. Jurgens UJ, Drews $G$, Weckesser J. Primary structure of the peptidoglycan from the unicellular cyanobacterium Synechocystis sp. strain PCC 6714 J Bacteriol. 1983;154:471-8.

20. Hoiczyk E, Hansel A. Cyanobacterial cell walls: news from an unusual prokaryotic envelope. J Bacteriol. 2000;182:1191-9.

21. Trautner C, Vermaas WFJ. The sll1951 gene encodes the surface layer protein of Synechocystis sp. strain PCC 6803. J Bacteriol. 2013;195:5370-80.

22. Heidelberg JF, Nelson WC, Schoenfeld T, Bhaya D. Germ warfare in a microbial mat community: CRISPRs provide insights into the co-evolution of host and viral genomes. PLoS ONE. 2009:4:e4169.

23. Jensen $H B$, Kleppe $K$. Effect of ionic strength, $\mathrm{pH}$, amines and divalent cations on the lytic activity of T4 lysozyme. Eur J Biochem. 1972;28:116-22.

24. Porter J, Mehigh R. Method for extracting a target product from a host cell employing zwitterionic detergent combinations. 2011.

25. Shin M. Ferredoxin-NADP reductase from spinach. Methods Enzymol. 1971;23:440-7

26. Cappellaro C, Baldermann C, Rachel R, Tanner W. Mating type-specific cell-cell recognition of Saccharomyces cerevisiae: cell wall attachment and active sites of a- and alpha-agglutinin. EMBO J. 1994;13:4737-44.

27. Perry $L$, Wetzel R. Disulfide bond engineered into T4 lysozyme: stabilization of the protein toward thermal inactivation. Science. 1984;226:555-7.

28. Gibson DG. Enzymatic assembly of overlapping DNA fragments. Methods Enzymol. 2011:498:349-61.

29. Kuchenreuther JM, Stapleton JA, Swartz JR. Tyrosine, cysteine, and S-adenosyl methionine stimulate in vitro [FeFe] hydrogenase activation. PLoS ONE. 2009;: 4 e7565.

30. Golden SS, Brusslan J, Haselkorn R. Genetic engineering of the cyanobacterial chromosome. Methods Enzymol. 1987;153:215-31.

31. Ivleva NB, Golden SS. Protein extraction, fractionation, and purification from cyanobacteria. Methods Mol Biol. 2007;362:365-73.

\section{Submit your next manuscript to BioMed Central and take full advantage of:}

- Convenient online submission

- Thorough peer review

- No space constraints or color figure charges

- Immediate publication on acceptance

- Inclusion in PubMed, CAS, Scopus and Google Scholar

- Research which is freely available for redistribution 